\title{
Materiales Digitales para Fortalecer el Aprendizaje Disciplinar en Educación Media Superior: Un Estudio para Comprender cómo se Suscita el Cambio Educativo
}

\author{
Digital Materials to Strength Learning Process in Upper \\ Secondary Education: A Study to Understand how Educational \\ Change takes Place
}

\author{
Katherina Edith Gallardo Córdova ${ }^{1 *}$ \\ Marta Araceli Alvarado García ${ }^{1}$ \\ Armando Lozano Rodríguez ${ }^{2}$ \\ Claudia Susana López Cruz ${ }^{1}$ \\ Sandra Gudiño Paredes ${ }^{1}$
}

${ }^{1}$ Tecnológico de Monterrey ${ }^{2}$ Centro Regional de Formación Docente e Investigación Educativa

\begin{abstract}
Se presenta un estudio en el marco de un proceso de cambio educativo en relación con la técnica Aula Invertida con apoyo de la herramienta de aprendizaje Living Class (LC) para apoyar el estudio de las disciplinas de química y ciencias sociales en educación media superior. El proceso de cambio se define a partir de dos aportaciones teóricas que integran cinco pasos: iniciación, planeación, implantación, evaluación e institucionalización. Se realizaron tres sub-estudios para indagar a profundidad el proceso de cambio que se suscitaba en la institución: uno sobre usabilidad de la herramienta LC y dos sobre percepción alrededor del proceso de cambio y apoyo en la herramienta, los cuales se enfocaron en colectar la percepción de docentes y estudiantes respectivamente. Participaron en total 4,708 estudiantes y 154 docentes. El estudio se enmarcó en el diseño metodológico de Métodos mixtos. Las conclusiones conllevaron a afirmar que el proceso de cambio educativo se encuentra en la fase de implantación. La discusión apunta a diagnosticar el estadio en el que se encuentra un proceso de cambio educativa de forma holística. Se realizan sugerencias para estudios futuros.
\end{abstract}

Descriptores: Cambio, Aprendizaje, Recursos educativos abiertos, Educación formal, Educación básica.

This article presents the results of a study made in order to analyze a process of educational change in upper secondary level. It focused on the implementation of Flipped Classroom technique supported by a learning tool called Living Class (LC). The educational change phenomena is defined within this study from two theoretical contributions that build a five steps process: initiation, planning, implementation, evaluation and institutionalization. Three sub-studies were conducted to investigate in depth the process of educational change that is experimented in the institution: one on LC usability and two others on students and teacher's perception about the target change process. A total of 4,708 students and 154 professors participated in this study. The methodological design was based on Mixed Methods. After the analysis, it is concluded that the target process is located by now in the implementation phase. The discussion points out the importance of diagnose an educational change phenomenon in a holistic way. Some suggestions for future studies are given.

Keywords: Change, Learning, Open educational resources, Formal education, Basic education.

*Contacto: katherina.gallardo@itesm.mx

ISSN: $1696-4713$

www.rinace.net/reice/

revistas.uam.es/reice
Recibido: $\quad 16$ de mayo 2016

$1^{\text {a }}$ Evaluación: 23 de agosto 2016

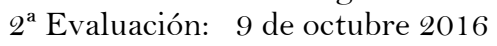

Aceptado: 13 de diciembre 2016 


\section{Introducción}

Las tendencias educativas en torno al uso de recursos educativos abiertos (REA) y objetos digitales de aprendizaje (ODA) experimentan un crecimiento considerable en los diferentes niveles educativos (Glasserman y Montoya, 2014; Morales Morgado, 2013; Solano Guerrero, 2012). La UNESCO (2014) impulsa una serie de iniciativas en países como México, Canadá, Inglaterra y Nueva Zelanda para apoyar el Movimiento de consulta y uso de recursos educativos abiertos (MEA). Posicionar el MEA de forma global podría ser la opción educativa que ayudaría a reducir la brecha entre la falta de educación y la posibilidad de desarrollar una serie de habilidades esenciales para el desempeño en el campo laboral y el crecimiento personal. Sin embargo, se sabe que una serie de recursos abiertos aislados podrían, por sí mismos, no impactar en el aprendizaje como se esperaría. En algunas ocasiones es preciso realizar un proceso de selección cuidadoso, acompañado de un diseño instruccional apropiado para que los recursos educativos abiertos cobren mayor significado para el aprendiz (Rivera, López Ibarra y Ramírez Montoya, 2011; Santos-Hermosa, Ferran-Ferrer y Adabal, 2012). Dentro de las tendencias educativas, el uso de las tecnologías de información y de las comunicaciones (TIC) ha sido constante y cada vez más frecuente en los distintos niveles educativos. Tradicionalmente, la educación mediada por tecnología e internet ha recurrido a herramientas estandarizadas o de desarrollo propio con la finalidad de facilitar la interacción entre docentes y alumnos. Dichas herramientas, ofrecen un entorno controlado en el cual se pueden colocar elementos y funciones, objetos de aprendizaje o herramientas de retroalimentación (Coates, James y Baldwin, 2005).

Existen, en la actualidad, una serie de iniciativas basadas en el uso y difusión de objetos digitales de aprendizaje para fortalecer el proceso educativo en torno a ciertas disciplinas o al desarrollo de alguna habilidad en especial: matemáticas, ciencias naturales, química, un segundo idioma, entre otros intereses disciplinarios. No obstante, la tendencia de construir repositorios o de diseñar objetos digitales pocas veces ha sido motivo de investigación en cuanto a su relación con el fenómeno del cambio educativo (Hueros, 2000); es por esta razón que los procesos de investigación que llevarían a entender los avances de estas iniciativas a la luz de la teoría del cambio educativo son escasas.

En los últimos 10 años se han producido alrededor de 40 artículos relacionados con el uso de materiales digitales educativos en Redalyc del 2005 a la fecha. En esta misma ventana de tiempo se hizo una búsqueda en Scielo, pudiendo hallarse 15 artículos publicados. No obstante, ninguna de estas investigaciones realizadas fue concebida a la luz de la teoría del cambio educativo.

Por lo anterior, la presente investigación tuvo como finalidad conocer el proceso de cambio en una institución educativa de nivel medio superior que busca implementar la técnica denominada Aula Invertida o Flipped Classroom por su denominación en inglés con la finalidad de propiciar el aprendizaje transformador, partiendo del bagaje del educando y de la cultura de la institución como requisitos indispensables para generar y sostener el cambio (Hutchings y Quinney, 2015). Esta investigación buscó comprender no sólo las implicaciones de diseño, de uso y los resultados obtenidos, sino el proceso de cambio educativo que subyace a la iniciativa, tanto desde la perspectiva de los docentes como de los estudiantes. 


\section{Fundamentación teórica}

Las bases epistemológicas y empíricas que fundamentan esta investigación se relacionan con: (1) la teoría del cambio educativo; (2) innovaciones educativas basadas en tecnología y uso de materiales digitales de aprendizaje; y (3) cambios en el proceso enseñanzaaprendizaje en el nivel medio superior: el Aula Invertida como una técnica para responder a necesidades educativas actuales.

\subsection{Teoría del cambio educativo}

El cambio educativo es, según Fullan y Stiegelbauer (2009) el proceso de transformación en el ámbito formativo que se presenta en tres etapas: (1) iniciación; (2) implementación; y (3) institucionalización. Murillo y Krichesky (2012) complementan esta definición y proceso con dos fases intermedias: (a) panificación, ubicada entre iniciación e implementación; y (b) evaluación, entre implementación e institucionalización. La figura 1 ilustra las aportaciones de Fullan y Stiegelbauer (2009) y Murillo y Krichesky (2012).

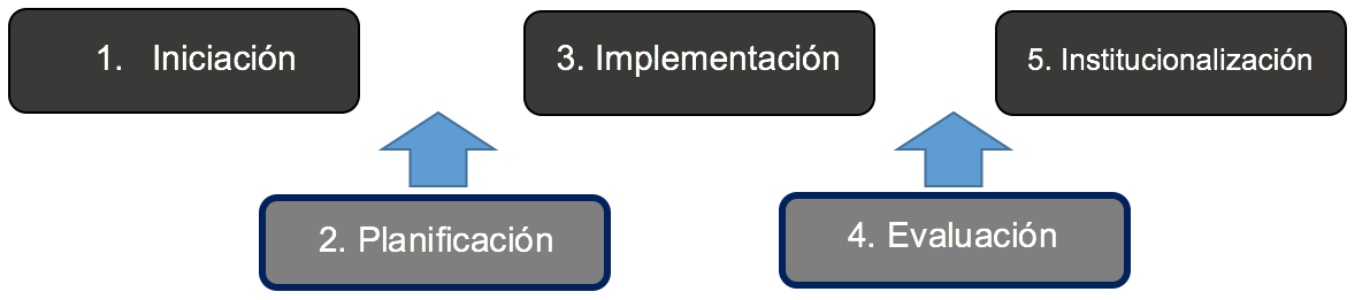

Figura 1. Fases que conforman el proceso de cambio educativo Fuente: Elaboración propia a partir de Fullan y Stiegelbauer (2009) y Murillo y Krichesky (2012).

Con base en el orden del proceso de cambio en el que se fundamenta este estudio (figura 1) se realizan las siguientes definiciones:

La fase de iniciación: detona el proceso de cambio en la cual interactúan ocho factores, de los cuales se toman tres para fines de este artículo: (a) innovaciones educativas; (b) los medios de comunicación; e (c) interacción efectiva entre docentes. Los participantes idóneos para esta fase son aquellos maestros con mayor actualización disciplinar y tecnológica, con alto nivel de eficacia, altas habilidades de colaboración y una franca actitud de compañerismo (Fullan y Stiegelbauer, 2009). A manera de complemento, Murillo y Krichesky (2012) agregan algunos elementos más a esta fase: (d) establecimiento de objetivos de manera conjunta colegiada; (e) generación de un sentido de motivación compartida; (f) diseminación del sentido de voluntad para generar el cambio; y (g) impulso a una cultura de mejora continua.

La fase de planeación: se refiere al momento dedicado al cuidado de aspectos técnicos del proceso de cambio en aras de convertir los deseos o las intenciones de mejora en acciones concretas. Esta fase se vincula con los objetivos a alcanzar en el proceso de mejora y, por consiguiente, en la elección y dosificación de estrategias en el tiempo. Aunque su diseño es una de las claves para el éxito del proceso de cambio, es pertinente velar también por su calidad técnica y el grado de consenso que se genere entre toda la comunidad. 
La fase de implementación: consiste en llevar a la práctica la idea o proyecto. Algunas interrogantes que deben formularse en esta etapa son: $\dot{c} 1$ ué cambiaría si se implementara ese proceso? ¿están alumnos y docentes dispuestos a modificar prácticas, convicciones e incluso hacer uso de otros materiales? Fullan y Stiegelbauer (2009) recomiendan recompensas tempranas y cierto éxito tangible que motive a continuar trabajando en el cambio. Se debe realizar una definición clara de las necesidades que se pretende cubrir con el proceso de cambio y en las que cada participante buscará satisfacer sus propias necesidades.

Las variables involucradas en esta fase son: (a) la complejidad, relacionada con la dificultad y extensión del cambio; (b) la claridad y factibilidad del programa, referido a la congruencia entre objetivos y acciones evitando así ansiedad y/o frustración entre los involucrados; y (c) una planeación evolutiva en la que se aproveche cualquier evento fortuito para ir adaptando. De hecho, los elementos que forman parte de un proceso de cambio exitoso son:

- Propiciar espacios de socialización para compartir prácticas exitosas

- Promover el apoyo continuo

- Infundir entusiasmo e inspiración

- Permitir la experimentación

- Capacitar apropiadamente

- Asignar recursos

- Brindar asistencia continua y oportuna durante la implementación

- Fomentar reuniones regulares con colegas

- Implementar una supervisión continua y solucionar problemas en donde la evaluación y seguimiento serán elementos vitales

- Tener claridad en objetivos y medios de implementación así como un adecuado diseño de la visión, que apunta hacia la dirección del cambio

Murillo y Krichesky (2012) complementan lo anterior advirtiendo que algunos teóricos sugieren trabajar en una fase previa denominada preimplementación, cuyo propósito sería preparar una implementación exitosa.

La fase de evaluación: A partir del establecimiento de una serie de indicadores es posible colectar información cuantitativa y cualitativa en torno al nivel de impacto de los cambios que se esperan en la práctica. Por lo anterior, es importante definir cuáles serán las evidencias que permitirán saber si se han alcanzado los resultados esperados acorde con la naturaleza del cambio. Esta fase implica el establecimiento de criterios comunes. A la vez, se requiere el diseño de instrumentos según los objetivos planteados. Estos pueden ser: cuestionarios, entrevistas, análisis de bases de datos, revisión de informes, etc.

La fase de institucionalización: Fullan y Stiegelbauer (2009) afirman que un proceso de institucionalización exitoso es generador de cambios desde la estructura organizacional lo que desencadenará un impacto directo hacia el interior de su cultura. Un proceso 
positivo en este rubro se distingue por la voluntad y el compromiso existente entre los implicados para lograr el cambio consistentemente, permitiendo su vivencia en la cotidianidad. Cada proceso de innovación no solo debe mantenerse sino también diseminarse. Algunos aspectos que afectan la viabilidad y continuidad del proceso referido son:

- Informalidad en relación con el apoyo financiero

- Capacitación deficiente

- Falta de compromiso de parte de los docentes

- Frecuente rotación de personal administrativo y escolar

\subsection{Innovaciones educativas basadas en tecnología y uso de materiales digitales de aprendizaje}

Las tendencias de habilitación masiva de recursos para la enseñanza, en conjunción con las nuevas tendencias encaminadas con el uso de herramientas Web 2.0, han puesto a disposición de millones de usuarios y en diferentes idiomas una serie de materiales educativos en formato digital para atender necesidades de formación en disciplinas diversas (Díaz et al., 2015; Suárez et al., 2015; Vidal Ledo et al., 2013). Las políticas mundiales han ido tomando fuerza rápidamente a partir de la flexibilidad y diálogo que se presentó en la UNESCO en el año 2002. Se decidió iniciar discusiones sobre el concepto y uso de Objetos o Recursos Educativos Abiertos. La institución sostiene desde entonces foros internacionales de diálogo sobre el tema con el afán de compartir ideas en favor de la cooperación internacional y mejores formas de aprovechar dichos recursos de aprendizaje (Díaz et al., 2015).

Sin duda, la opción de tener acceso a recursos generados con intenciones de formación en formato de acceso abierto aumenta a la disponibilidad del material a públicos diversos en cualquier parte del globo. Sin embargo, las opciones de aprovechamiento de los recursos se potencializan cuando éstos se encuentran agrupados intencionalmente bajo criterios de cohesión y afinidad, ya sea por disciplinas, temas de interés o habilidades que se pretenden desarrollar. A esta agrupación de recursos educativos abiertos se les denomina repositorios (Santos-Hermosa, Ferran-Ferrer y Abadal, 2012). Otra variedad de estos recursos se encuentra en la opción de diseñar materiales digitales con intenciones específicas cuando en el banco de recursos no se encuentran materiales hechos a la medida. A estos objetos de aprendizaje diseñados con fines específicos y que se vinculan con recursos de libre acceso se les denomina objetos digitales de aprendizaje (Morales Morgado, 2013; Solano Guerrero, 2012).

En materia de TIC y uso de materiales digitales, es también relevante destacar la tendencia que los últimos años que ha ido marcando preferencias de uso y planteamiento de retos en los ambientes educativos a favor de lograr un cambio. Al respecto, se insiste en que una variable importante en torno a entender el cambio educativo a la luz de las TIC es la tendencia a usar un determinado artefacto, servicio o material. Además, el tiempo de adopción es otra de las variables relevantes a considerar en este tema. Ante la diversidad y la premura por apropiarse de las nuevas tecnologías, es frecuentemente 
difícil dar continuidad a los proyectos con fines de lograr y evaluar los logros en torno a los cambios en el proceso educativo.

La tabla 1 muestra una indagación realizada por el grupo de investigadores de Fischer y colaboradores (2015) en relación con lo declarado en los Reportes Horizon (elaborado por New Media Consortium y Educause) sobre las tendencias en uso de TIC, lo cual podría ayudar a aclarar el panorama en torno a las tendencias y el tiempo de adopción de las innovaciones que han revolucionado el mundo de la educación en los últimos años.

No obstante la variabilidad en TIC y materiales digitales que marquen tendencias en la innovación y el cambio, resulta importante resaltar la labor de los docentes en la selección y conducción de estas iniciativas. Ellos son finalmente sobre quienes recae la responsabilidad de diseñar, operacionalizar y entregar estrategias educativas basadas en el uso de materiales digitales así como los resultados de su intervención, que vislumbren el grado en que un cambio esperado ha sido logrado.

Celaya Ramírez, Lozano Martínez y Ramírez Montoya (2010) realizaron un estudio sobre cinco casos de docentes que integraron a su práctica formativa el uso de materiales digitales clasificados como recursos educativos abiertos (REA). El análisis cualitativo realizado giró en torno al conocimiento, uso, aplicación en contextos diferentes, experiencia del docente y proceso de adopción. A través de las entrevistas, cuestionarios y revisión de documentos sobre su planeación didáctica se emitieron resultados en torno a esta integración, de los cuales los principales resultados fueron: (1) se detectó que la gran mayoría de los docentes perciben que con el uso de estos materiales apoyaron su proceso de enseñanza, porque utilizaron estímulos visuales que emplearon como medio para promover la interactividad y la creatividad; (2) el uso de estos materiales aparentemente incide en la mejor comprensión de temas abstractos dado que se trabajó con simuladores y gráficos para clarificar el significado de los elementos estudiados; (3) el uso de estos materiales detonaron en los preparatorianos sentido de iniciativa y colaboración.

\subsection{Cambios en el proceso enseñanza-aprendizaje en el nivel medio superior: el} Aula Invertida como una técnica para responder a necesidades educativas actuales

El nivel medio superior ha sido uno de los niveles que más modificaciones ha sufrido en los últimos años, tanto en México como en otros países de América Latina. En el marco de las reformas educativas recientes, el cambio de paradigma hacia el avance de constituir y operacionalizar el Modelo Educativo Basado en Competencias (MEBC) en este nivel ha demandado trabajar en una estrategia nacional de formación docente. En el caso de México, dentro de las metas de formación contenidas en la reforma educativa para media superior, se encontró la necesidad de formar a los docentes en la inserción de tecnología en el aula (Gamboa y Maldonado, 2012; SEMS, 2014).

Una constante en los países de América Latina es la búsqueda de alternativas para que los resultados de la educación media superior se vean reflejados en elevar los índices de la matrícula en educación superior y se modernicen los procesos con uso de TIC (Reyes, Patrón y Argüelles, 2015; Sánchez, 2015). Para lograrlo, uno de los factores clave es contar con hábitos de estudio y gusto por la lectura. 
Tabla 1. Tendencias en el uso de TIC y tiempos de adopción a lo largo de siete años (2007-2014)

\begin{tabular}{|c|c|c|c|c|c|c|c|c|}
\hline & 2014 & 2013 & 2012 & 2011 & 2010 & 2009 & 2008 & 2007 \\
\hline \multirow[t]{2}{*}{1 año } & $\begin{array}{c}\text { Aula } \\
\text { invertida }\end{array}$ & MOOC & $\begin{array}{l}\text { Disp. móviles } \\
\text { APPs }\end{array}$ & Disp. móviles & Disp. móviles & Disp. móviles & Videos básicos & $\begin{array}{l}\text { Contenido } \\
\text { creado por } \\
\text { usuarios }\end{array}$ \\
\hline & $\begin{array}{l}\text { Analíticas de } \\
\text { aprendizaje }\end{array}$ & Tabletas & Tabletas & $\begin{array}{c}\text { Libros } \\
\text { electrónicos }\end{array}$ & $\begin{array}{l}\text { Acceso abierto a } \\
\text { contenido }\end{array}$ & $\begin{array}{c}\text { Computación en } \\
\text { la nube }\end{array}$ & $\begin{array}{l}\text { Colaboración } \\
\text { por Internet }\end{array}$ & Redes sociales \\
\hline \multirow{2}{*}{ 2-3 años } & Impresión 3D & $\begin{array}{c}\text { Juegos y } \\
\text { gamificación }\end{array}$ & $\begin{array}{l}\text { Aprendizaje } \\
\text { basado en } \\
\text { juegos }\end{array}$ & $\begin{array}{l}\text { Aprendizaje } \\
\text { basado en } \\
\text { juegos }\end{array}$ & $\begin{array}{l}\text { Libros } \\
\text { electrónicos }\end{array}$ & Geo-todo & $\begin{array}{c}\text { Ancho de } \\
\text { banda móvil }\end{array}$ & $\begin{array}{l}\text { Teléfonos } \\
\text { móviles }\end{array}$ \\
\hline & $\begin{array}{c}\text { Juegos y } \\
\text { gamificación }\end{array}$ & $\begin{array}{l}\text { Analíticas de } \\
\text { aprendizaje }\end{array}$ & $\begin{array}{l}\text { Analíticas de } \\
\text { aprendizaje }\end{array}$ & $\begin{array}{l}\text { Realidad } \\
\text { aumentada }\end{array}$ & $\begin{array}{l}\text { Realidad } \\
\text { aumentada } \\
\text { simple }\end{array}$ & La Web personal & $\begin{array}{c}\text { Datos } \\
\text { combinados }\end{array}$ & $\begin{array}{l}\text { Mundos } \\
\text { virtuales }\end{array}$ \\
\hline \multirow{2}{*}{ 4-5 años } & $\begin{array}{c}\text { Ser } \\
\text { cuantificable }\end{array}$ & Impresión 3D & $\begin{array}{l}\text { Computación } \\
\text { basada en } \\
\text { gestos }\end{array}$ & $\begin{array}{l}\text { Computación } \\
\text { basada en } \\
\text { gestos }\end{array}$ & $\begin{array}{l}\text { Computación } \\
\text { basada en } \\
\text { gestos }\end{array}$ & $\begin{array}{c}\text { Aplicaciones con } \\
\text { consciencia } \\
\text { semántica }\end{array}$ & $\begin{array}{l}\text { Inteligencia } \\
\text { colectiva }\end{array}$ & $\begin{array}{l}\text { La nueva } \\
\text { colegiatura }\end{array}$ \\
\hline & $\begin{array}{l}\text { Asistente } \\
\text { virtual }\end{array}$ & $\begin{array}{l}\text { Tecnología } \\
\text { para vestir }\end{array}$ & $\begin{array}{l}\text { El internet de } \\
\text { las cosas }\end{array}$ & $\begin{array}{l}\text { Analíticas de } \\
\text { aprendizaje }\end{array}$ & $\begin{array}{c}\text { Análisis de } \\
\text { datos de forma } \\
\text { visual }\end{array}$ & $\begin{array}{c}\text { Objetos } \\
\text { inteligentes }\end{array}$ & $\begin{array}{l}\text { Sistemas de } \\
\text { operación } \\
\text { social }\end{array}$ & $\begin{array}{l}\text { Ed. basada en } \\
\text { juegos con } \\
\text { multi-jugadores }\end{array}$ \\
\hline
\end{tabular}

Fuente: Fischer y sus colaboradores (2015). Traducción al español por autores del artículo. 
Dentro de las múltiples alternativas para dinamizar y modernizar los procesos de enseñanza-aprendizaje en educación media superior, se encuentra la opción de la técnica denominada Aula Invertida como una técnica que podría facilitar el uso del tiempo áulico en procesos de enseñanza-aprendizaje que apunten hacia el logro de mayor profundidad en el estudio de los contenidos disciplinares.

$\mathrm{Al}$ definir la técnica Aula Invertida, se puede afirmar que es un método pedagógico que emplea una serie de materiales de uso asincrónico como videos o problemas de práctica para su resolución fuera del aula. La selección y dosificación de materiales para su estudio se realiza de forma anticipada. La construcción previa a la sesión de clases permite apropiarse de los contenidos que se reafirman en el aula. A su vez, permite al maestro dedicar más tiempo al trabajo con problemas o ejercicios más complejos que demanden procesos de pensamiento más elevados, preferentemente en el marco del trabajo en equipo (García-Barrera, 2013; Tucker, 2012). Así, a través de la técnica Aula Invertida se generará no solo la adquisición del conocimiento, sino también de competencias transversales tales como: toma de decisiones, trabajo colaborativo, poder de negociación, intercambio cultural, planeación estratégica, capacidad para debatir y ser mediadores, entre otros. Esta técnica se fundamenta sobre una base socioconstructivista. Esta base teórica propicia el que cada miembro obtenga un beneficio colectivo incluso en ejercicios de reflexión y conceptualización.

Una de las implicaciones más importantes de esta técnica es el cambio del papel del alumno. Así, el estudiante se concibe como el mayor responsable de su formación de manera directa, teniendo en mente que deberá prepararse con antelación en horarios fuera de clase. De hacerlo así, se podrá tener una exitosa participación dentro del aula, elevando las posibilidades de alcanzar con mayor rapidez prácticas autónomas de aprendizaje. Diversos estudios, en el área de medicina por ejemplo, han comprobado la eficacia en cuanto a la elevación de la responsabilidad, la eficiencia y por ende en el rendimiento académico (Abeysekera y Dawson, 2015; Tune, Sturek y Basile, 2013). Adicionalmente, aplicar Aula Invertida puede ser una adecuada decisión didáctica para los jóvenes, dado que esta técnica les brinda a los estudiantes libertad de interactuar con el contenido de acuerdo con su estilo de aprendizaje (Roehl, Reddy y Shannon, 2013).

En cuanto al papel del docente, la función esencial es planear el trabajo previo del alumno y ser un constante detonador de procesos constructivos. Por tanto, su liderazgo intelectual se mantiene vital también en esta metodología. Es preciso mencionar en este punto, la ineludible presencia de la tecnología a través de la cual los objetos de aprendizaje cobran significado debido a que serán elementos de apoyo para enriquecer la dinámica del aula invertida (Angelini y García-Carbonell, 2015).

\section{Problematización}

Se decidió diseñar una estrategia educativa con base en el uso de materiales digitales combinando ODA y REA, para apoyar un proceso de cambio educativo relacionado con la inserción de la técnica Aula Invertida en las materias de química y ciencias sociales de tercer semestre de educación media superior. La estrategia educativa inició con el diseño de un libro electrónico y evolucionó con el paso del tiempo a la conformación de un sitio web donde se aloja el material digital intencionalmente diseñado y seleccionado por expertos en las disciplinas antes mencionadas. Su denominación es Living Class (LC). La 
pregunta de investigación es ¿En qué estadio se encuentra el proceso de cambio educativo relacionado con la adopción de la técnica Aula Invertida?

\section{Método}

\section{Diseño}

Este estudio se realizó desde un enfoque de Métodos mixtos (Johnson y Onwuegbuzie, 2004; Valenzuela y Flores, 2012) dada la necesidad de comprender de manera profunda el proceso de cambio educativo que pudo haberse experimentado a partir de la utilización de LC como una estrategia diseñada con el fin de fomentar la instauración de la técnica denominada Aula Invertida. Dada la escasa producción científica en relación con el tema se decidió diseñar este estudio bajo los parámetros de un estudio exploratorio.

La investigación se realizó a partir de tres sub-estudios: (1) usabilidad del recurso LC, (2) uso de LC como herramienta didáctica por parte de los docentes, y (3) uso de LC como herramienta de aprendizaje por parte de los estudiantes. La justificación subyace a que se requirió indagar cada uno de estos factores para poder analizar el proceso de cambio e identificar en el estadio en el que se encuentra la estrategia de forma holística.

\section{Contexto}

Este estudio se llevó a cabo en una institución educativa privada que opera con un total de 30 campus en diferentes Estados de la República Mexicana. En todos ellos se utiliza LC como material auxiliar de las materias Química y Ciencias Sociales. LC contiene una serie de referencias bibliográficas, lecturas, videos, juegos interactivos y materiales multimedia, el cual fue diseñado por docentes expertos en las disciplinas mencionadas.

LC se puso a disposición de todos los docentes y estudiantes del tercer semestre de preparatoria por lo que se creó un acceso a este material para toda la comunidad educativa y se colocó en la plataforma o aula virtual. El 100\% la población docente y estudiantil del tercer semestre tuvo acceso a este repositorio durante el periodo agostodiciembre 2015.

\section{Participantes e instrumentación}

Los participantes en el estudio fueron tanto docentes como alumnos que sostienen actividad académica en el tercer semestre del plan de estudios de bachillerato y que interactuaron con la herramienta LC durante el periodo lectivo mencionado anteriormente. Para cada sub-estudio los investigadores diseñaron instrumentos que les permitirían colectar información en relación con los objetivos propuestos. La tabla 2 conjunta los objetivos de cada sub-estudio, los participantes e instrumentos para cada uno de los sub-estudios.

\section{Procedimiento}

Se realizaron un total de tres sub-estudios para entender a profundidad las acciones alrededor de la estrategia de usar LC para propiciar el cambio deseado: (1) sub-estudio de usabilidad del recurso LC, (2) sub-estudio sobre el uso de LC como herramienta didáctica 
por parte de los docentes, y (3) sub-estudio sobre el uso de LC como herramienta de aprendizaje por parte de los estudiantes.

Tabla 2. Objetivo de cada sub-estudio, participantes e instrumentos utilizados por subestudio realizado e instrumentos utilizados

\begin{tabular}{|c|c|c|c|c|}
\hline & \multirow{2}{*}{ OBJETIVo } & \multicolumn{2}{|c|}{ PARTICIPANTES } & \multirow{2}{*}{ INSTRUMENTOS } \\
\hline & & Docentes & Estudiantes & \\
\hline $\begin{array}{c}\text { Sub-estudio } \\
1 . \\
\text { Usabilidad } \\
\text { de LC }\end{array}$ & $\begin{array}{l}\text { Conocer en qué } \\
\text { medida, la usabilidad } \\
\text { de la herramienta LC } \\
\text { resultó óptima para } \\
\text { enriquecer la práctica } \\
\text { del docente y del } \\
\text { alumno. El grado de } \\
\text { usabilidad se estimó a } \\
\text { partir de cinco } \\
\text { criterios: facilidad de } \\
\text { aprendizaje, eficiencia, } \\
\text { memorabilidad, } \\
\text { número margen de } \\
\text { errores y satisfacción } \\
\text { (Nielsen, 1993) }\end{array}$ & $\begin{array}{l}\text { N: } 330 \\
5 \text { desarrolladores } \\
\text { (profesores que } \\
\text { diseñaron LC en } \\
\text { cuanto al diseño } \\
\text { de materiales y } \\
\text { lugar que debe } \\
\text { ocupar los } \\
\text { materiales en la } \\
\text { navegación) } \\
5 \text { novatos } \\
5 \text { habituados }\end{array}$ & $\begin{array}{l}\text { N: } 7.096 \\
14 \text { novatos } \\
14 \\
\text { habituados }\end{array}$ & $\begin{array}{l}\text { Lista de } \\
\text { actividades básica } \\
\text { ( } 75 \text { en total) } \\
\text { Observación a } \\
\text { través del registro } \\
\text { en video de la } \\
\text { interacción de } \\
\text { usuarios } \\
\text { categorizados de } \\
\text { acuerdo a su } \\
\text { experiencia }\end{array}$ \\
\hline $\begin{array}{c}\text { Sub-estudio } \\
2 . \\
\text { Uso de LC } \\
\text { por parte de } \\
\text { los docentes }\end{array}$ & $\begin{array}{l}\text { Indagar si los docentes } \\
\text { que emplean LC son } \\
\text { más competentes en el } \\
\text { uso de la tecnología y } \\
\text { si son más } \\
\text { innovadores en su } \\
\text { práctica educativa }\end{array}$ & $\begin{array}{l}139 \text { encuestados } \\
20 \text { entrevistados } \\
31 \text { en grupos } \\
\text { focales }\end{array}$ & & $\begin{array}{l}\text { Cuestionario } \\
\text { Entrevista } \\
\text { individual } \\
\text { Grupo focal }\end{array}$ \\
\hline $\begin{array}{l}\text { Sub-estudio } \\
\qquad 3 . \\
\text { Uso de LC } \\
\text { por parte de } \\
\text { los } \\
\text { estudiantes. }\end{array}$ & $\begin{array}{l}\text { Identificar si los } \\
\text { alumnos reconocen el } \\
\text { uso de los recursos } \\
\text { que contiene LC como } \\
\text { un factor que favorece } \\
\text { su aprendizaje } \\
\text { Identificar si existe } \\
\text { alguna diferencia en el } \\
\text { rendimiento } \\
\text { académico entre } \\
\text { grupos de estudiantes } \\
\text { que utilizaron los } \\
\text { recursos de LC en } \\
\text { diferentes campus } \\
\text { durante el periodo } \\
\text { agosto-diciembre } 2015\end{array}$ & & $\begin{array}{l}4.680 \\
\text { encuestados } \\
62 \text { grupos } \\
\text { focales } \\
17 \\
\text { entrevistados }\end{array}$ & $\begin{array}{l}\text { Cuestionario } \\
\text { Entrevista } \\
\text { individual } \\
\text { Grupo focal }\end{array}$ \\
\hline
\end{tabular}

Fuente: Elaboración propia.

En el sub-estudio sobre usabilidad las fases trabajadas fueron las siguientes:

- Selección y revisión de actividades por parte de desarrolladores y equipo de evaluación y medición de impacto

- Selección aleatoria de participantes por parte de los centros educativos

- Registro en video de las sesiones de trabajo con la plataforma en cada uno de los centros escolares y aplicación de entrevistas semiestructuradas. 
- Extracción y procesamiento de datos

- Resultados e interpretación

Participaron en el estudio 49 personas de diferentes campus: 14 alumnos novatos en el uso de LC, 14 alumnos habituados al uso de LC docentes, 11 profesores habituados, 5 profesores novatos y 5 docentes que fueron desarrolladores de la herramienta.

En el segundo y tercer sub-estudio se contó con la participación de docentes y estudiantes en diferentes proporciones según necesidades de indagación (ver tabla 2). En todos los casos, se preparó el proceso de participación, primero con mensajes vía electrónica: se enviaron comunicados masivos a toda la población docente y estudiantil para la aplicación del cuestionario. Posteriormente, se seleccionaron al azar docentes y estudiantes para proceder con las entrevistas individuales y para la realización de grupos focales. Cabe resaltar que en el caso de las entrevistas se utilizaron casi al $100 \%$ sistemas remotos de videoconferencia para su registro.

Se realizaron cinco grupos focales con docentes y alumnos. En el caso de los docentes, se contó con la participación de 31 de ellos provenientes de diferentes campus. También se llevaron a cabo siete grupos focales con alumnos, todos ellos de manera presencial. Se contó con la participación de 62 estudiantes en total.

Una vez terminadas las aplicaciones se procedió al procesamiento de datos: las respuestas del cuestionario de docentes y estudiantes (139 y 4.680 respectivamente) se ingresaron en SPSS para su análisis e interpretación. En el caso de las transcripciones de grupos focales y entrevistas, se analizaron utilizando la herramienta Atlas.ti.

\section{Resultados}

Se exponen a continuación los resultados de tres sub-estudios hechos sobre el uso de LC como herramienta diseñada para fomentar la práctica del Aula Invertida en las materias de Química y Ciencias sociales. Posteriormente, se interpretan estos resultados a la luz del proceso de cambio educativo que se aspira a lograr a partir de la teoría de cambio educativo y sus fases tal como lo señalan Fullan y Stiegelbauer (2009) y Murillo y Krichesky (2012).

\subsection{Sub-estudio sobre usabilidad}

Los resultados obtenidos de este primer sub-estudio permitieron estimar qué tanto la herramienta LC podría ser utilizada apropiadamente desde el punto de vista tecnológico. Así, se emitieron los resultados con base en cinco criterios que al medirse estiman en su conjunto el nivel de usabilidad de herramientas electrónicas (Nielsen, 1993). Estos factores son: (1) facilidad de aprendizaje; (2) eficiencia; (3) memorabilidad; (4) margen de errores; y (5) satisfacción.

- Facilidad de Aprendizaje: los resultados de las pruebas permitieron apreciar que a los alumnos y docentes categorizados como novatos, o sin experiencia previa de uso de LC les tomó mayor tiempo realizar las 75 actividades dentro de la plataforma respecto a docentes y alumnos que ya tenían experiencia previa. Sin embargo, el tiempo de diferencia entre ambas categorías generales 
fue de 14.5 segundos. Por tanto se infiere que LC es una herramienta fácil de usar, inclusive para los usuarios novatos que requieren resolver las tareas asignadas cuando se encuentran con esta herramienta por primera vez. Respecto a la función de usuarios como docentes y alumnos, los resultados permitieron confirmar el cumplimiento de este criterio, específicamente en los docentes novatos dado que los integrantes de este grupo concluyeron las actividades en menor tiempo que los docentes con experiencia previa. La diferencia de tiempo fue de 14,11 segundos respecto a los expertos.

- Eficiencia: este criterio permitió medir qué tan rápido los usuarios pueden resolver una serie de tareas de navegación y acceso a materiales. Los resultados obtenidos derivaron en aseverar que LC cumple con este criterio dada la cantidad de actividades por minuto que cada una de las categorías de usuarios con experiencia previa logró completar. En promedio, el tiempo en completar las actividades fueron de 3.01 segundos para docentes expertos y 1.98 segundos para alumnos expertos. La figura 2 contiene los detalles de estos resultados

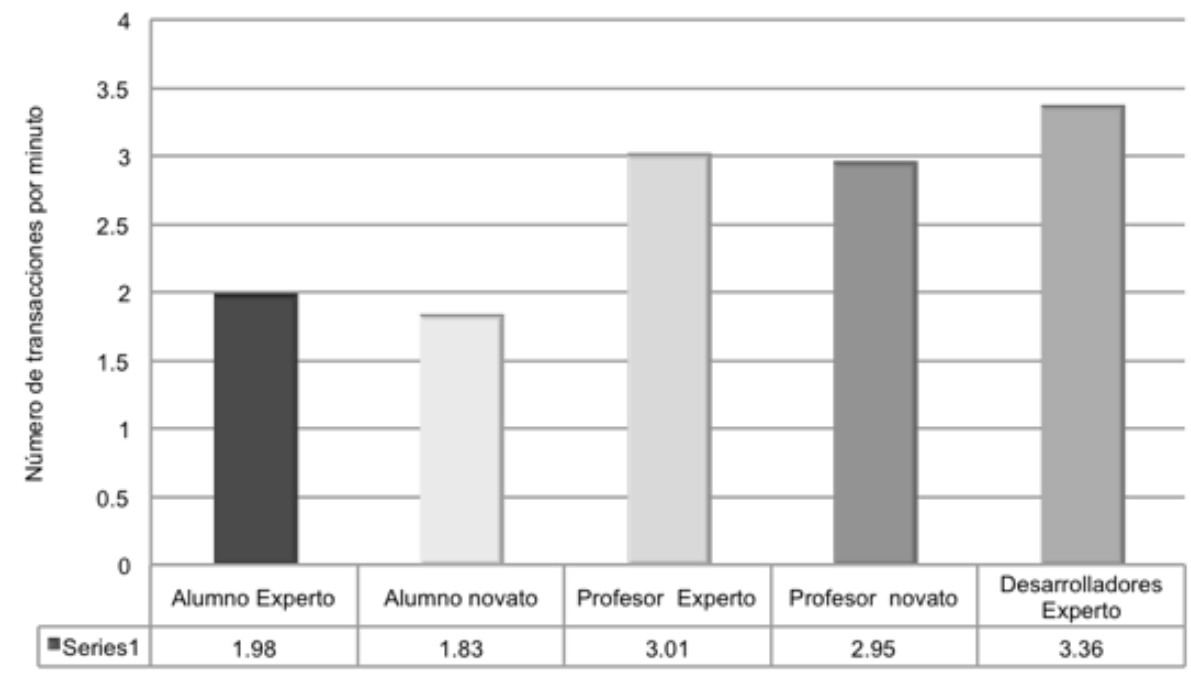

Figura 2. Resultados a partir de la medición del criterio de eficiencia de LC Fuente: Elaboración propia.

- Memorabilidad: a partir de este criterio se midió la facilidad con que los usuarios podrían restablecer las competencias ya adquiridas para resolver tareas con uso de LC. Los resultados apuntaron a que a los usuarios les resultó fácil restablecer las competencias adquiridas en actividades previas para completar aquellas subsecuentes. Se destaca que al grupo de alumnos novatos les llevó una mayor cantidad de tiempo restablecer las competencias que a otros grupos.

- Margen de error: a partir de este criterio se pudo detectar cuáles actividades generaron mayor cantidad de errores por parte de los usuarios durante la navegación y uso de herramientas en LC. La ejecución de las 75 tareas permitió 
hacer una estimación del porcentaje de errores de los diferentes grupos de usuarios. Los resultados se muestran en la tabla 3.

Tabla 3. Tasa de error calculada en el proceso de evaluación de usabilidad de LC en las diferentes categorías de usuarios

\begin{tabular}{lc}
\hline \multicolumn{1}{c}{ CATEGORÍA } & TASA DE ERROR \\
\hline Alumnos expertos & $9 \%$ \\
Alumnos novatos & $1 \%$ \\
Profesores novatos & $5 \%$ \\
Profesores expertos & $1 \%$ \\
Desarrolladores & $0.1 \%$ \\
\hline
\end{tabular}

Fuente: Elaboración propia.

Satisfacción: las entrevistas permitieron entender que el grado de satisfacción por parte de los usuarios es alto. Se realizaron declaraciones de usuarios novatos en las que utilizaron las palabras "fácil” y "sencilla" de forma recurrente.

\subsection{Sub-estudio sobre uso de LC por parte de docentes}

Este sub-estudio se enfocó en indagar si los docentes que utilizaron LC han experimentado, desde su percepción, algún nivel de desarrollo en sus competencias sobre el uso de la tecnología, su capacidad innovadora, cambios en su estilo didáctico, en el proceso de adopción de la técnica Aula Invertida y el uso del tiempo en el aula, entre las principales. El cuestionario utilizado obtuvo una alta consistencia interna (0,93 Alfa de Cronbach). La figura 3 muestra los resultados.

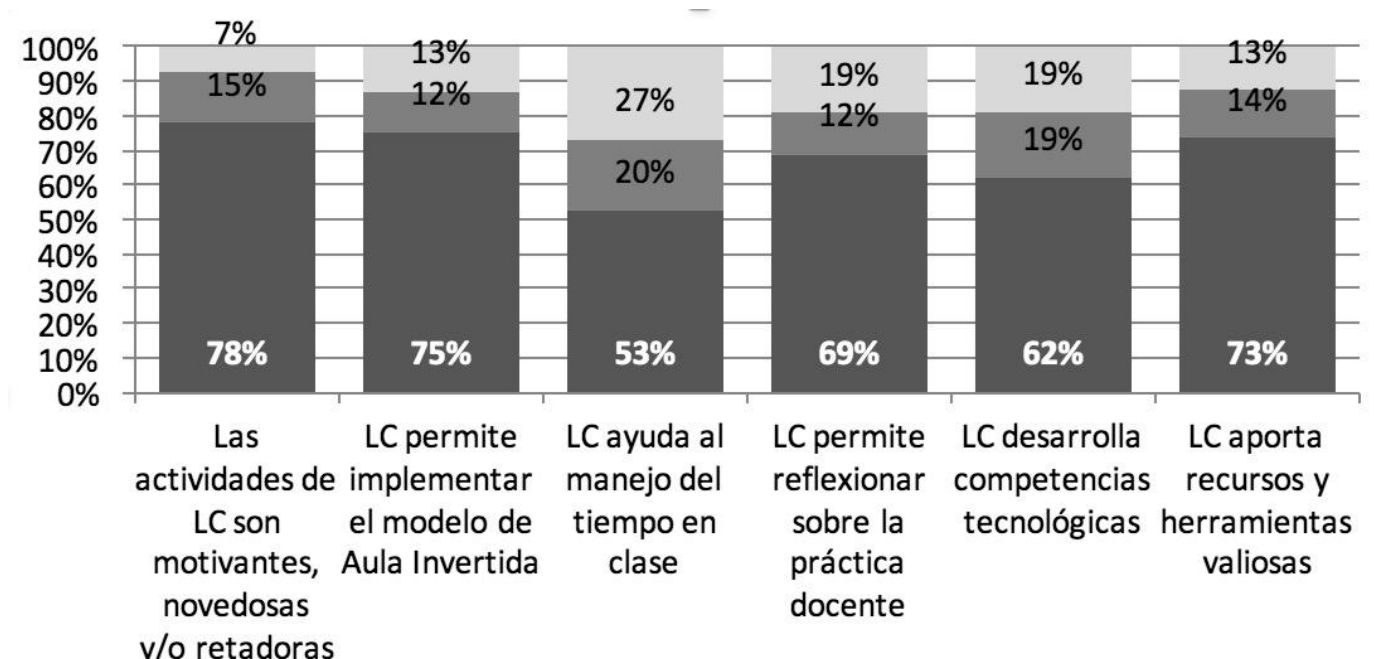

\section{De acuerdo En desacuerdo Indiferente}

Figura 3. Principales resultados de la encuesta a docentes que usan LC Fuente: Elaboración propia.

Los docentes encuentran motivantes las actividades que se incluyen en LC, novedosas y retadoras en un alto porcentaje (78\%). Esto coincide con el hecho de que opinan que LC aporta recursos y herramientas valiosas (73\%). Asimismo, perciben que el uso de LC ha 
sido satisfactorio para implementar la técnica del Aula Invertida (75\%). Sin embargo, en un porcentaje menor (62\%) perciben que LC coadyuva a desarrollar sus competencias en el uso de tecnología. Cabe destacar que en las entrevistas los docentes declararon una serie de aspectos vinculados con el desarrollo de competencias tecnológicas orientadas a la formación y sobre su relación con el éxito que esto podría derivar en la aplicación de la técnica Aula Invertida. En especial los profesores novatos mencionaron que dentro de los aprendizajes logrados con el uso de LC como herramienta didáctica, perciben cambios relacionados con la integración de tecnología en su práctica docente, lo cual favorece aprovechar mejor su tiempo en el aula para actividades más complejas. A continuación se enlistan las principales ideas expuestas por los docentes:

- Manejo de aplicaciones para gamificación: WordDrop, Crosswords, Kahoot y Piktochart

- Interés por la exploración intuitiva de nuevas herramientas.

- Vinculación de contenidos teóricos con la vida real.

- Ejercicio del pensamiento creativo para integrar más recursos y actividades al aula acorde con intereses de los estudiantes.

- No obstante, en las entrevistas los docentes también manifestaron preocupaciones y sugerencias en relación con el uso de LC.

- Carencia de herramientas que permitieran conducir autoevaluaciones de aprendizaje.

- Falta de ejercicios que permitan mayor interacción y respuestas inmediatas.

- Carencia de foros donde llevar a cabo discusiones en tiempo real.

- Requerimiento de más actividades lúdicas o gamificadas.

\subsection{Sub-estudio sobre uso de LC por parte de estudiantes}

Este sub-estudio se enfocó en aspectos relacionados con el apoyo de LC, tanto en el tiempo áulico como en el de estudio independiente. El cuestionario utilizado obtuvo una alta consistencia interna (0,94 Alfa de Cronbach). La figura 4 permite apreciar que LC es un recurso que más del $50 \%$ de los estudiantes lo considera útil para reforzar conceptos y temas además de ser utilizado para fines de estudio para presentar exámenes.

En cuanto a las preguntas que se relacionan directamente con el proceso de estudio independiente usando LC, requisito indispensable para poder aplicar la técnica de Aula Invertida, se aprecia que los estudiantes en un $42,35 \%$ afirmaron tener prácticas de estudio independiente bajo estas características. Además, se realizó un análisis para entender si existe alguna correlación entre la percepción del alumno sobre su rendimiento académico y el uso de LC para haber logrado o mejorado las calificaciones que ha obtenido en las materiales en las que se usa la herramienta. El resultado fue positivo en tanto se confirmó significatividad en la correlación mencionada. Se presenta la figura 5 con estos resultados. 

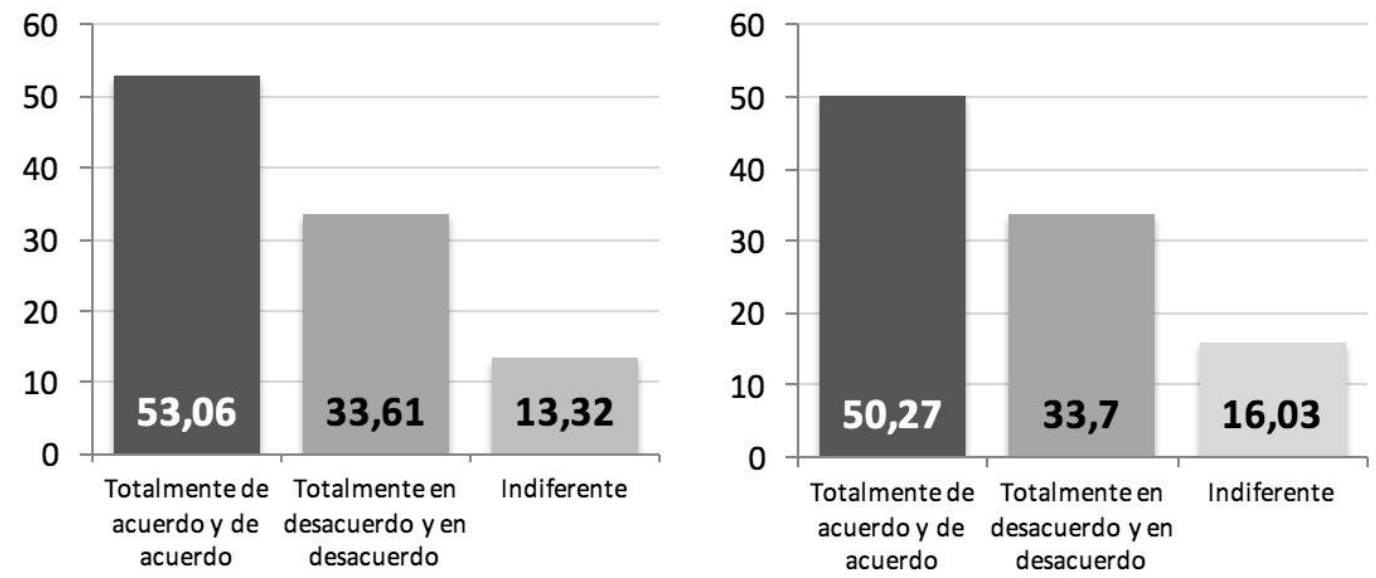

Figura 4. Ayuda de LC para clarificar o reforzar conceptos y temas (izquierda). Utilidad de LC para estudiar para los exámenes (derecha)

Nota: Valores expresados en porcentajes. Total de datos procesados: 4.397. No contestan: 283.

Fuente: Elaboración propia.
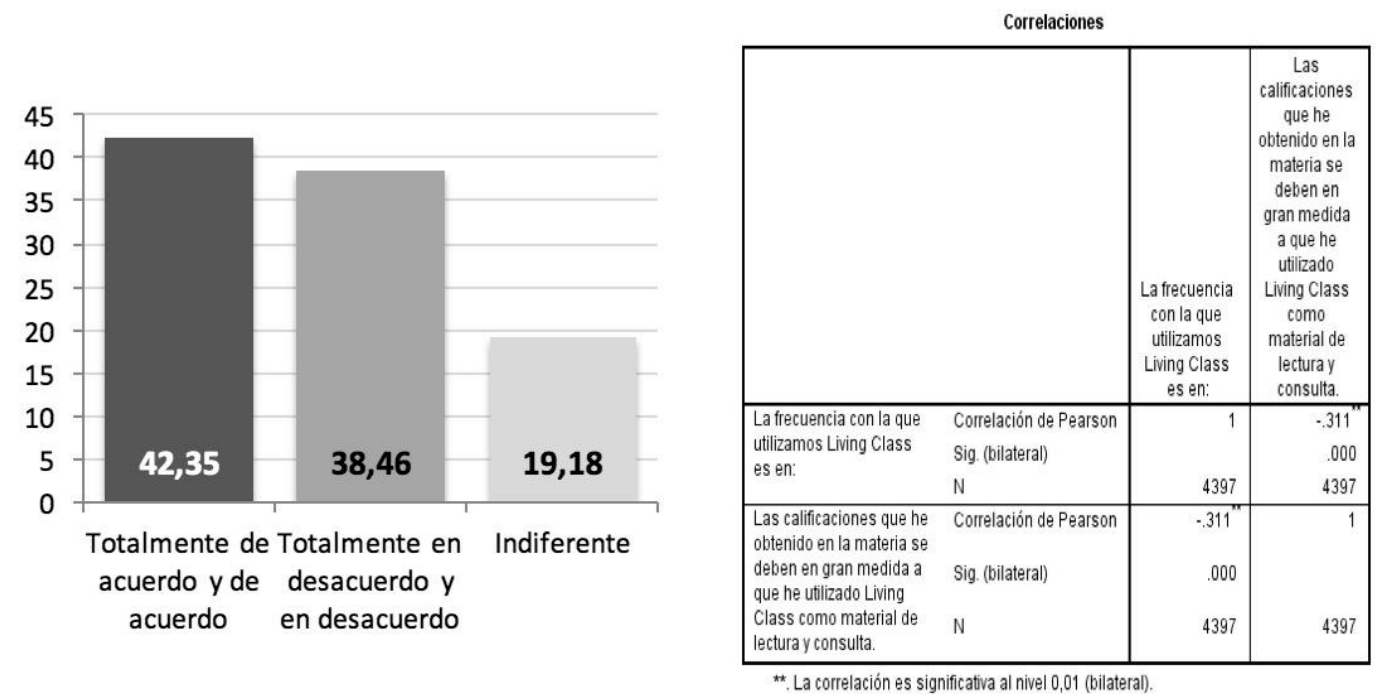

Figura 5. Estudio de materiales contenidos en LC por cuenta propia conforme lo exige la técnica Aula Invertida (izquierda) y resultados de la correlación entre percepción de uso de LC con rendimiento académico (derecha)

Nota: Valores expresados en porcentajes. Total de datos procesados: 4.397. No contestan: 283.

Fuente: Elaboración propia.

Por último, cabe destacar que las entrevistas permitieron terminar de entender algunas apreciaciones de los estudiantes sobre el uso de LC en el marco de la técnica Aula Invertida. Expresaron ideas que se pueden resumir de la siguiente forma:

- Se describieron diferentes prácticas sobre cómo el docente introdujo LC al estudiante el primer día de clases. Algunos docentes sí realizaron una introducción formal y detallada. Otros mencionaron vagamente LC en clase, 
haciéndolo de forma general y sin hacer alusión a su relación con la técnica Aula Invertida

- Existe disparidad en cuanto al tiempo de uso de LC dentro y fuera del aula así como la frecuencia semanal con la que se hace en las materias donde se utiliza (química y ciencias sociales).

- Las solicitudes hechas por los docentes sobre el estudio de los materiales de LC de forma independiente (fuera del aula) se concentran más en tiempos de exámenes

\section{Discusión y conclusiones}

A partir de los resultados obtenidos en los tres subestudios y en función de la problematización de esta investigación, se ha diseñado la tabla 4. El objetivo es presentar las ideas más relevantes en torno a lo que cada subestudio aportó para comprender dónde y por qué se podría afirmar que el proceso de cambio en la institución de estudio se encuentra en una determinada fase. Sin duda, cada uno de los hallazgos en contraste con la teoría y el estado del arte permiten indicar el nivel a lo largo del proceso

Este estudio buscó entender en qué fase del proceso de cambio educativo se encuentra un esfuerzo institucional al pasar de una dosificación tradicional de actividades en el aula hacia el uso de la técnica Aula Invertida con el fin de aprovechar el tiempo de interacción alumnos-profesor para trabajar en actividades que demanden mayor complejidad y generen más conocimiento. En este caso, se concluye que aunque la herramienta LC se encuentra en una fase de evaluación, el proceso formativo se encuentra en la fase de implementación.

A partir de lo anterior, se infiere que en este caso en particular el proceso de cambio no subyace aun en una definición clara por parte del grueso de población sobre el fin último de esta estrategia educativa. Se espera que en esta fase aún se presenten dificultades como la complejidad relacionada con la extensión del cambio y la comunicación congruente y alineada entre objetivos y acciones, entre las principales (Fullan y Stiegelbauer, 2009). Así, las percepciones de alumnos y profesores alrededor del uso de LC se presentan muy poco orientados hacia el fin último de este esfuerzo educativo. Esta aseveración recae en las opiniones muy marcadas sobre el uso para la práctica áulica y temas vinculados como el estudio para exámenes, el tiempo de uso en clase y, en contraste, la poca expresión del uso de LC para fines de la implementación del Aula Invertida. Es decir, aunque en este caso LC se encuentre consolidado y en fase de evaluación, se requiere analizar los factores centrales del proceso formativo antes de aseverar que por el hecho de contar con una herramienta tecnológica a punto de entrar en la fase de institucionalización, en la misma etapa se encuentre todo el proceso que el cambio educativo involucra.

Por los resultados presentados, se estima que la fase de implementación continuará su curso. Es posible que a lo largo de ella se logren abrir más espacios de socialización, permitir la experimentación con algunas variables que se vislumbren necesarias, lograr fortalecer la comunicación colegiada, etc. Así, finalmente se podría trabajar de manera 
conjunta y organizada hacia el logro de la implementación de la técnica con apoyo de LC y otras estrategias que permitan el fortalecimiento de esta práctica educativa.

Tabla 4. Discusión en función del proceso de cambio educativo a partir de los resultados de los tres subestudios

\begin{tabular}{|c|c|c|}
\hline $\begin{array}{c}\text { SUB- } \\
\text { ESTUDIOS }\end{array}$ & DISCUSIÓN EN TORNO AL ESTUDIO & $\begin{array}{c}\text { FASE DEL PROCESO DE CAMBIO EN LA } \\
\text { QUE SE UBICA }\end{array}$ \\
\hline $\begin{array}{l}\text { Usabilidad } \\
\text { de LC }\end{array}$ & $\begin{array}{l}\text { Al evaluar los criterios de usabilidad, } \\
\text { los resultados permiten inferir } \\
\text { cumplimiento satisfactorio reflejado } \\
\text { en el tiempo estimado de la } \\
\text { interacción con LC que demandó cada } \\
\text { tarea, contrastando el desempeño de } \\
\text { docentes y alumnos expertos y } \\
\text { novatos (Nielsen, 1993). }\end{array}$ & $\begin{array}{l}\text { Fase de evaluación: se aprecia a LC como } \\
\text { un conjunto de materiales digitales } \\
\text { agrupados intencionalmente para apoyar } \\
\text { el proceso de aprendizaje de las materias } \\
\text { de ciencias naturales y sociales que } \\
\text { brinda sin dificultades procesos de } \\
\text { navegación, exploración, consulta de } \\
\text { ODA y REA sin mayor contratiempo, ya } \\
\text { sea para usuarios expertos o novatos. El } \\
\text { estudio de los criterios de usabilidad } \\
\text { funge como el proceso de evaluación. }\end{array}$ \\
\hline $\begin{array}{c}\text { Uso de LC } \\
\text { por parte de } \\
\text { docentes }\end{array}$ & $\begin{array}{l}\text { La percepción general de los } \\
\text { profesores lleva a calificar a LC como } \\
\text { un recurso satisfactorio y de ayuda } \\
\text { para implementar la técnica Aula } \\
\text { Invertida, aunque el tiempo para } \\
\text { dosificar las actividad áulicas todavía } \\
\text { no está del todo dominado. Además, } \\
\text { se han estimulado algunas otras } \\
\text { competencias en el uso de TIC. Sin } \\
\text { embargo, se aprecia una serie de } \\
\text { carencias que podrían afectar el uso } \\
\text { idóneo de la herramienta (Angelini y } \\
\text { García-Carbonell, 2015). }\end{array}$ & $\begin{array}{l}\text { Fase de implementación: a través de las } \\
\text { respuestas se aprecia que el uso de LC } \\
\text { inicia actividades encaminadas hacia un } \\
\text { proceso de inserción de la técnica Aula } \\
\text { Invertida. Se infiere a partir de los } \\
\text { resultados que los docentes están } \\
\text { realizando procesos de aplicación, } \\
\text { experimentación, descubrimiento de sus } \\
\text { capacidades e innovación en su práctica } \\
\text { docente, todo orientado a provocar el } \\
\text { cambio. No obstante, no se aprecia un } \\
\text { fenómeno generalizado de apropiación } \\
\text { del fin último del uso de LC. }\end{array}$ \\
\hline $\begin{array}{l}\text { Uso de LC } \\
\text { por parte de } \\
\text { estudiantes }\end{array}$ & $\begin{array}{l}\text { En el caso de los estudiantes, su } \\
\text { percepción apunta hacia dos prácticas } \\
\text { recurrentes: se está utilizando LC } \\
\text { como una herramienta de apoyo } \\
\text { aunque con prácticas diversas en } \\
\text { cuanto a dosificación de tiempo en } \\
\text { clase, frecuencia semanal y modo de } \\
\text { uso (dentro y fuera del aula). Las } \\
\text { opiniones en las encuestas y su } \\
\text { complemento en entrevistas lleva a } \\
\text { inferir que existe todavía camino por } \\
\text { recorrer para homologar más los } \\
\text { criterios de uso así como las } \\
\text { consignas para que sea realmente una } \\
\text { herramienta que coadyuve a la } \\
\text { práctica del Aula Invertida (García- } \\
\text { Barrera, 2013; Roehl, Reddy y } \\
\text { Shannon, 2013; Tucker, 2012) }\end{array}$ & $\begin{array}{l}\text { Fase de implementación: Las } \\
\text { aseveraciones de los estudiantes llevan a } \\
\text { evaluar que el proceso de cambio se } \\
\text { encuentra iniciando este tercer estadio. } \\
\text { Los estudiantes tienen en claro que el } \\
\text { uso de LC tiene como objetivo apoyar el } \\
\text { proceso de enseñanza-aprendizaje pero } \\
\text { no así claridad en que lo que se quiere } \\
\text { finalmente lograr es la inserción del Aula } \\
\text { Invertida como técnica. Tampoco se } \\
\text { aprecia que los estudiantes estén } \\
\text { participando en proceso de evaluación } \\
\text { regular de la herramienta. Sus opiniones } \\
\text { se centran más en que es una } \\
\text { herramienta vinculada a tener éxito en } \\
\text { los exámenes que en propiamente en } \\
\text { fomentar una cultura del estudio } \\
\text { anticipado a la clase. }\end{array}$ \\
\hline
\end{tabular}

Fuente: Elaboración propia.

Para futuros estudios, se sugiere seguir indagando en situaciones similares donde el proceso de cambio educativo se geste con base en estrategias académicas y tecnológicas, pudiendo privilegiar mayor conocimiento sobre este fenómeno. 


\section{Referencias}

Abeysekera, L. y Dawson, P. (2015). Motivation and cognitive load in the flipped classroom: definition, rationale and a call for research. Higher Education Research \& Development, 34(1), 1-14. doi:10.1080/07294360.2014.934336

Angelini, M. L. y García-Carbonell, A. (2015). Percepciones sobre la integración de modelos pedagógicos en la formación del profesorado: La simulación y juego y el flipped classroom. Teoría de la Educación. Educación y Cultura en la Sociedad de la Información, 16(2), 16-30. doi:10.14201/eks20151621630

Celaya Ramírez, R., Lozano Martínez, F. y Ramírez Montoya, M. S. (2010). Apropiación tecnológica en profesores que incorporan recursos educativos abiertos en educación media superior. Revista Mexicana de Investigación Educativa, 15(45), 487-513.

Coates, H., James, R. y Baldwin, G. (2005). A critical examination of the effects of learning management systems on university teaching and learning. Tertiary Education and Management, 11, 19-36. doi:10.1080/13583883.2005.9967137

Díaz, J., Schiavoni, A., Amadeo, A. P., Charnelli, M. E., Schulz, J. G. y Humar, A. (2015). Integrando un repositorio digital de objetos de aprendizaje con servicios que promuevan su uso y mantenimiento. Conferencias LACLO, 5(1), 1-24.

Fischer, H., Heise, L., Heinz, M., Moebius, K. y Koehler, T. (2015). How to identify e-learning trends in academic teaching. Interactive Technology and Smart Education, 12(1), 31-56.

Fullan, M. G. y Stiegelbauer, S. (2009). El cambio educativo: Guía de planeación para maestros. Ciudad de México: Trillas.

Gamboa, L. F. y Maldonado, D. (2012). Análisis de la evolución de la igualdad de oportunidades en educación media, en una perspectiva internacional. El caso de Colombia. En M. Peña (Dir.), Estudios sobre la calidad de la educación en Colombia (pp. 43-67). Bogotá: ICFES

García-Barrera, A. (2013). El aula inversa: Cambiando la respuesta a las necesidades de los estudiantes. Avances en Supervisión Educativa, 19, 1-8.

Glasserman, L. y Montoya, M. S. (2014). Uso de recursos educativos abiertos (REA) y objetos de aprendizaje (OA) en educación básica. Teoría de la Educación, Educación y Cultura en la Sociedad de la Información, 15(2), 86-102.

Hueros, A. M. D. (2000). Innovación y nuevas tecnologías: implicaciones para un cambio educativo. En-clave Pedagógica, 2, 129-145.

Hutchings, M. y Quinney, A. (2015). The flipped classroom, disruptive pedagogies, enabling technologies and wicked problems: responding to "the bomb in the basement". Electronic Journal of E-Learning, 13(2), 106-119.

Johnson, R. B. y Onwuegbuzie, A. (2004). Mixed methods research: A research paradigm whose time has come. Educational Research, 33(7), 14-26. doi: 10.3102/0013189x033007014

Morales Morgado, E. M. (2013). Desarrollo de competencias a través de objetos de aprendizaje. RED Revista de Educación a Distancia, 36, 1-24,

Murillo, F. J. y Krichesky, G. J. (2012). El proceso de cambio escolar: una guía para impulsar y sostener la mejora de las escuelas. REICE. Revista Iberoamericana sobre Calidad, Eficacia y Cambio en Educación 10(1), 27-43.

Nielsen, J. (1993). Usability engineering. Boston, MA: AP Professional. 
Reyes, P. C., Patrón, R. M. y Argüelles, L. A. (2015). Mejora de las competencias tecnológicas del docente en la educación media superior. Revista Iberoamericana para la Investigación y el Desarrollo Docente, 1(1), art. 3.

Rivera, R. López Ibarra, A. y Ramírez Montoya, M. S. (2011). Compartiendo conocimiento: estrategias de comunicación para potenciar el uso de recursos educativos abiertos para procesos de enseñanza innovadores. Recuperado de http://www.ruv.itesm.mx/

Roehl, A., Reddy, S. L. y Shannon, G. J. (2013). The flipped classroom: An opportunity to engage millennial students through active learning. Journal of Family and Consumer Sciences, 105(2), 44-72.

Sánchez, S. M. (2015). Educación en la nube. Un nuevo reto para los docentes de educación media superior. Revista Iberoamericana para la Investigación y el Desarrollo Educativo, 10, 288-301.

Santos-Hermosa, G., Ferran-Ferrer, N. y Abadal, E. (2012). Recursos educativos abiertos: repositorios y uso. El Profesional de la Información, 21(2), 136-145. doi:10.3145/epi.2012.mar.03

SEMS. (2014). Infografías sobre la reforma educativa en México. Recuperado de http://www.sems.gob.mx/en_mx/sems/infografias_reforma_educativa_ems

Solano Guerrero, J. C. (2012). Modelos de representación de conocimiento en el diseño de objetos digitales de aprendizaje. Revista Perspectivas Educativas, 5, 101-122.

Suárez, A. I., Pérez Rodríguez, C. Y., Vergara Castaño, M. M. y Alférez Jiménez, V. H. (2015). Desarrollo de la lectoescritura mediante TIC y recursos educativos abiertos. Apertura, 7(1), $1-13$.

Tucker, B. (2012). The flipped classroom. Education Next, 12(1), 1-24.

Tune, J. D., Sturek, M. y Basile, D. P. (2013). Flipped classroom model improves graduate student performance in cardiovascular, respiratory, and renal physiology. Advances in Physiology Education, 37(4), 316-320. doi:10.1152/advan.00091.2013

UNESCO. (2014). Guía básica de recursos educativos abiertos. Recuperado de http://static1.squarespace.com/static/5 1ede959e4bode4b8d24e8a9/t/564e4cebe4bo7e1b 9ab3fo53/1447972075739/Recursos+Educativos+Abiertos+REA+\%28UNESCO\%29.pdf

Valenzuela, J. R. y Flores, M. (2012). Fundamentos de la investigación educativa. Monterrey: Editorial Digital del Tecnológico de Monterrey.

Vidal Ledo, M. J., Alfonso S. I. y Zacca González, G. y Martínez Hernández, G. (2013). Recursos educativos abiertos. Educación Médica Superior, 27(3), 307-320.

\section{Breve CV de los autores}

\section{Katherina Edith Gallardo Córdova}

Docente e investigadora de la Escuela de Educación, Humanidades y Ciencias Sociales del Tecnológico de Monterrey. Es miembro del Sistema Nacional de Investigadores avalado por el CONACyT (Nivel 1). Tiene estudios de doctorado en Innovación y Tecnología Educativa por el Tecnológico de Monterrey (ITESM). Dentro de sus funciones docentes, imparte cursos de evaluación del aprendizaje y tecnología e innovación en educación. Adicionalmente coordina un grupo de asesores tutores de tesis de maestría y ha dirigido varias disertaciones doctorales. Sus líneas de investigación 
giran alrededor de los temas relacionados con la solución de problemas y la evaluación del aprendizaje, entre las principales. Para acceder a los artículos publicados por esta autora, visitar: https://itesm.academia.edu/KatherinaGallardo ORCID ID: 0000-00018343-9518. Email: katherina.gallardo@itesm.mx

\section{Marta Araceli Alvarado García}

Licenciada en Sistemas de Computación Administrativa por el Tecnológico de Monterrey Campus Monterrey con Maestría en Educación con Especialidad en Cognición en los Procesos de Enseñanza Aprendizaje por la misma institución. Ha colaborado desde hace 20 años en el Tecnológico de Monterrey, de los cuales los últimos 15 lo ha hecho en la modalidad en línea diseñando cursos, planes de estudio, siendo profesora de profesional y maestría así como directora académica de profesores y del programa de profesional. Asimismo, participó activamente como miembro del equipo fundador de Universidad TecMilenio y del programa "Carreras por Internet". Actualmente es profesora de cátedra de la Escuela de Educación, Humanidades y Ciencias Sociales fungiendo además como lectora de tesis y sinodal en exámenes de grado. Con publicación de artículo en revista indizada titulado: Retroalimentación en línea: una estrategia para la construcción del conocimiento. ORCID ID: 0000-00023744-7709. Email: maalvara@itesm.mx

\section{Armando Lozano Rodríguez}

Docente e investigador del Centro Regional de Formación Docente e Investigación Educativa en Comitán de Domínguez, Chiapas. Es miembro del Sistema Nacional de Investigadores avalado por el CONACyT (Nivel 1). Tiene estudios de doctorado en Innovación y Tecnología Educativa por el Tecnológico de Monterrey (ITESM). Dentro de sus funciones docentes, imparte cursos de desarrollo de competencias, instrucción cognitiva, estilos de aprendizaje, tecnología educativa e innovación en educación. Adicionalmente coordina un grupo de asesores tutores de tesis de maestría y ha dirigido varias disertaciones doctorales. Sus líneas de investigación giran alrededor de los temas relacionados con estilos de aprendizaje, estilos de enseñanza, desarrollo de competencias y aplicaciones tecnológicas a la educación. Para acceder a los artículos publicados por este autor, visitar: https://itesm.academia.edu/ArmandoLozano ORCID ID: 0000-00027013-4210. Email: armando.lozano@cresur.edu.mx

\section{Claudia Susana López Cruz}

Doctora en Diseño, con una especialización en nuevas tecnologías y máster en Diseño con una concentración en Nuevas Tecnologías. Ambos posgrados otorgados por la Universidad Autónoma Metropolitana. Actualmente se desempeña como Líder Institucional de Experimentación y Medición de Impacto en el Tecnológico de Monterrey. Ha impartido a la fecha más de 50 cursos y talleres en los campos de educación tales como la metodología de la investigación, la formación en tecnología de la educación, la gestión de recursos y la creación de contenidos educativos abiertos. Asimismo, ha escrito una serie de obras y ha participado en congresos nacionales e 
internacionales con ponencias, entre las que destacan: Diseño de la biología o la biología de Diseño: las percepciones de la Ecología inmaterial; El aprendizaje en la fotografía digital; Las experiencias cognitivas de colaboración móvil y Movilidad en el aprendizaje: experiencias interdisciplinares con E-Learning, entre las principales. ORCID ID: 00000001-5205-9053. Email: clopez@itesm.mx

\section{Sandra Gudiño Paredes}

Licenciada en Administración y mercadotecnia por la Universidad Autónoma de Nuevo León. En 2012 concluyó la maestría en Tecnología Educativa en la Universidad Virtual del Tecnológico de Monterrey mientras se desempeñaba como docente de preparatoria. En el año 2013 logró la beca de investigación para estudiar el doctorado en innovación educativa en el tecnológico de Monterrey fungiendo como a la fecha como asistente de proyectos de investigación de tiempo completo auspiciados por organismos nacionales e internacionales. Ha publicado y editado artículos en revistas arbitradas e indizadas e impartido talleres y conferencias nacionales e internacionales relacionadas con su línea de investigación, la cual abarca temas de innovación educativa,bullying y desarrollo moral. Más sobre la autora: https://cinco-itesm.academia.edu/SandraGudi\%C3\%B1o. ORCID ID: 0000-0001-9786-606X. Email: sandra.guidno@itesm.mx 